Article

\title{
Nitric Oxide Generating Formulation as an Innovative Approach to Topical Skin Care: An Open-Label Pilot Study
}

\author{
Stephan Settelmeier ${ }^{1}$, Tienush Rassaf ${ }^{1}{ }^{(\mathbb{O}}$, Ulrike B. Hendgen-Cotta ${ }^{1}$ and Ingo Stoffels ${ }^{2, *}$ \\ 1 Department of Cardiology and Vascular Medicine, University Hospital Duisburg-Essen, Hufelandstr. 55, \\ 45147 Essen, Germany; stephan.settelmeier@uk-essen.de (S.S.); tienush.rassaf@uk-essen.de (T.R.); \\ ulrike.hendgen-cotta@uk-essen.de (U.B.H.-C.) \\ 2 Department of Dermatology, Venereology and Allergology, University Hospital Essen, Hufelandstr. 55, \\ 45147 Essen, Germany \\ * Correspondence: ingo.stoffels@uk-essen.de
}

check for updates

Citation: Settelmeier, S.; Rassaf, T.; Hendgen-Cotta, U.B.; Stoffels, I. Nitric Oxide Generating Formulation as an Innovative Approach to Topical Skin Care: An Open-Label Pilot Study. Cosmetics 2021, 8, 16. https:// doi.org/10.3390/cosmetics8010016

Academic Editor: Enzo Berardesca

Received: 29 December 2020

Accepted: 12 February 2021

Published: 16 February 2021

Publisher's Note: MDPI stays neutral with regard to jurisdictional claims in published maps and institutional affiliations.

Copyright: (c) 2021 by the authors. Licensee MDPI, Basel, Switzerland. This article is an open access article distributed under the terms and conditions of the Creative Commons Attribution (CC BY) license (https:// creativecommons.org/licenses/by/ $4.0 /)$.

\begin{abstract}
Nitric oxide (NO) plays multiple roles in both normal and abnormal skin processes. Its deranging disbalance is involved in the pathogenesis of multiple dermatologic diseases such as acne vulgaris, pointing towards beneficial therapeutic directions. A novel NO-producing gel-formulation was tested beneficial in the treatment of acne vulgaris in an open-label pilot study using clinical evaluation scores. It showed a decrease of comedones and inflammatory pustulae and reduced the Global Acne Grading System score by 50\% within eight weeks. In addition, we demonstrate a potential use as cosmetic agent where NO therapy leads to an increase of skin integrity and a reduction of skin ageing processes.
\end{abstract}

Keywords: nitric oxide; NO; acne vulgaris; topical therapy; skin ageing

\section{Introduction}

Nitric oxide (NO) is a diatomic molecule that plays many roles in both normal and abnormal skin processes [1,2]. There is strong evidence that NO-releasing materials may serve as new, unique therapeutic agents [3]. The skin's cell population consists of keratinocytes, endothelial cells, fibroblasts and other residing or circulating immune cell types. Nearly all of them express isoforms of nitric oxide synthase (NOS) enabling NO production which is essential for physiologic processes like antimicrobial defense, regulation of circulation as well as erythematic response to ultraviolet light exposure [3-5]. While endothelial NOS (eNOS) produces lower levels of NO, the inducible NOS (iNOS) produces larger amounts of NO when stimulated by e.g., bacterial products or cytokines [6]. NO is furthermore involved in creating the protective skin layer [3]. Therefore, NO is essential to maintain skin balance.

\subsection{Acne Vulgaris}

Facial acne vulgaris is the most common skin disease in adolescents and adults and a primary inflammatory disorder of the pilosebaceous unit of multifactorial etiology [7]. Four interrelated mechanisms are implicated in the development of acne vulgaris and responsible for underlying skin disbalance: increased sebum production, alterations of the follicular keratinization processes, inflammation and colonialization of Cutibacterium acnes (formerly Proprionibacterium acnes) [8]. With its long-lasting psychological negative effect, acne vulgaris has a negative impact on the patient's quality of life [9]. Treatment of acne reaches from mild topical cleaners to systemic treatment with antibiotic agents or antihormonal therapies, resulting in a therapeutic burden for many patients [7].

Within a physiological range, $\mathrm{NO}$ possesses a concentration-dependent antimicrobial and immunomodulatory bimodal activity [6]. However, using higher concentrations in 
topical application, NO demonstrates anti-inflammatory and antimicrobial properties, the latter without the risk of generating microbial resistance $[3,10]$.

\subsection{NO as a Cosmetic Agent}

Skin aging is, besides the biological age, dependent on the exposure to environmental factors, which affect the skin by structural and functional changes resulting in known characteristics like loss of elastic capacities, formation of wrinkles and loss of skin moisture [6]. Most of them being a target of cosmetic treatments. Due to its capability of restoring the skin barrier, NO application increases skin moisture [3] and possesses the capability of enhancing human collagen synthesis [4]. Due to its molecular size and distinct lipophilicity, NO itself can easily penetrate the outer layers of the skin but its potency is limited by its short half-life $[3,6]$. Therefore, different delivery platforms capable of stable release over a defined time period have been developed. Acidified nitrate cremes use acidic disproportionation for the release of $\mathrm{NO}$, diazeniumdiolates act as direct $\mathrm{NO}$-donators while direct application of gaseous NO or NO-releasing nanoparticles represent another way of application $[6,11]$. Nevertheless, the different application systems have limitations including the risk of methemoglobin formation on diazeniumdiolates, expensive and difficult release mechanisms in the case of gaseous formulation or technical issues concerning production and acceptance of nanoparticles. We therefore designed a novel NO-generating mutual-activating dual gel formulation and more fluid serum formulation with enhanced penetrative and nutritive properties which was examined for preliminary efficacy and proof of concept in mild to moderate acne (gel formulation) and as a cosmetic agent (serum formulation).

\section{Materials and Methods}

A novel NO-producing dual component mutual-activating gel formulation with $1 \%$ low molecular hyaluronic acid was examined for preliminary clinical efficacy on acne vulgaris in a single-center retrospective study with an open-label proof-of-concept design. The formulation was produced in pharmaceutical purity ( $>95 \%)$. The study was conducted in the Department of Dermatology, University Hospital Essen. Retrospective analysis of acne vulgaris patients was approved by the local ethics committee (20-9698-BO). For clinical evaluation as a cosmetic product agent, another single-center open study was conducted to assess the acceptability and efficacy of the investigational product after four weeks of use of the serum-formulation. Due to cosmetic product testing, ethics committee approval was not required. All subjects gave written informed consent for participation and publication. Histological analyses of $4 \mathrm{~mm}$-diameter intact upper arm skin were performed using hematoxylin and eosin (H\&E) staining. In-vitro $\left(1 \mathrm{~cm}^{3}\right.$ of each component) and on-skin $\left(7 \mathrm{~cm}^{2}\right.$ skin surface) NO-production capabilities were proofed using a nitric oxide chemiluminescent detector (CLD) [1,2].

\subsection{Patient Population}

Patients referring to our out-patients clinic for the treatment of acne vulgaris were evaluated for the study. Ten consecutive patients of both genders were included in the study. Inclusion criteria were: age between $18-50$ years and clinical diagnosis of mild to moderate acne, mainly located on the face. Each patient was instructed to apply a thin film of the two components topical gel as a monotherapy twice a day for 12 weeks. The two components were applied one after another and then mixed on the skin. Before topically applying the medication, patients were invited to thoroughly wash their facial skin using a gentle, nonmedicated cleanser, rinse with warm water and gently pat dry. Clinical and instrumental evaluations were performed at the beginning of the treatment (T0), after four weeks (T1) and after eight weeks (T2). 


\subsection{Proband Population}

The two components topical serum-formulation was tested in healthy volunteers. 11 women and four men (mean age 42 years) participated for clinical evaluation as a cosmetic agent. After four weeks of twice daily facial application, participants completed a questionnaire on the serum characteristics and effects. Skin samples were collected from the identically treated upper arm section.

\subsection{Efficacy and Safety Evaluation}

The main outcome of this study on acne was a change in the acne lesion number. Evaluation included the Global Acne Grading System (GAGS) to assess acne severity [12]. GAGS is a quantitative scoring system in which the final score is calculated by the sum of six regional subscores, which in turn derives by multiplying an established factor for each facial region [13]. Only patients suffering from mild to moderate facial were enrolled. Lesions count, through which major acne lesions are independently assessed, were comedons, papules/pustules and nodules/cysts [14]. The face was divided into several regions such as nose, forehead, each cheek, and perioral region. Photographic assessment was performed at each visit. The Dermatology Life Quality Index (DLQI) [15] was assessed at TO (beginning of treatment), T1 (4 weeks) and T2 (8 weeks). A questionnaire filled by the patients to assess the tolerability of the product (excellent, good, fair, poor) and the presence of side effects. For cosmetic evaluations, wrinkles, structural integrity and smoothing of the skin relief were evaluated using a full face imaging system (Observ 520, InnoFaith Beauty Sciences, Eindhoven, The Netherlands). Images and H\&E staining were evaluated visually by experienced dermatologists.

\section{Results}

\subsection{Measurement of NO-Generation}

Using the closed CLD system, an in vitro mixture of $1 \mathrm{~cm}^{3}$ of each component resulted in stable generation of $\mathrm{NO}$, initially exceeding $23.847 \mathrm{ppb}$, resulting in a plateau phase of $>15 \mathrm{~min}$ (Figure 1). NO generation was, although decreasing, still detectable $30 \mathrm{~min}$ after initial application. On-skin measurements on $7 \mathrm{~cm}^{2}$ intact skin showed comparable generation of NO.

\section{NO-generation of activated gel-formulation}

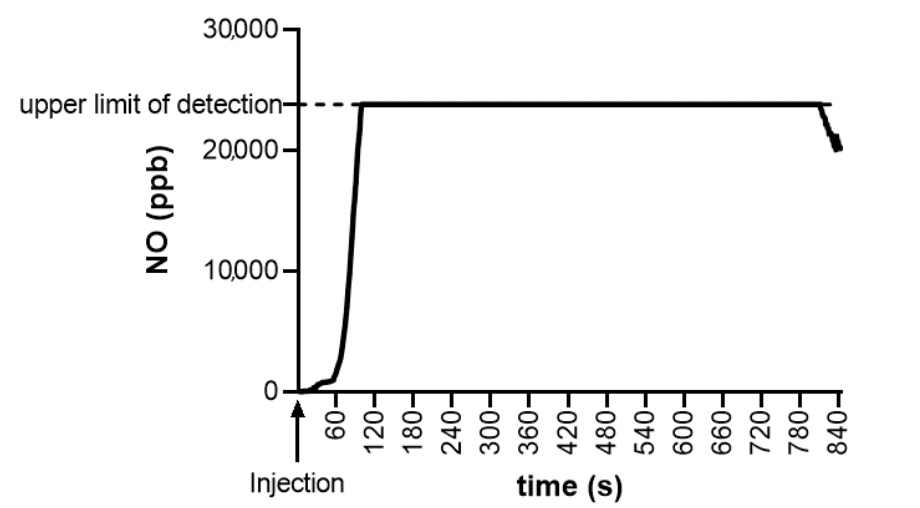

Figure 1. Nitric oxide (NO)-generation of activated gel-formulation. In vitro mixture of $1 \mathrm{~cm}^{3}$ of each component resulted in stable generation of $\mathrm{NO}$, exceeding the upper limit of detection of $23.847 \mathrm{ppb}$ $\mathrm{NO}$, resulting in a plateau phase of $>15$ min after activation time of approximately $60 \mathrm{~s}$.

\subsection{Clinical Evaluation of the Gel Formulation in Acne Vulgaris}

A total of 10 consecutive patients (one male and nine female) were enrolled in the study. Patients were assessed from June 2020 to October 2020. The mean age was 34.4 years. All patients completed the study and there were no drop-outs. The GAGS score showed a $32 \%$ reduction from $\mathrm{T} 0$ to $\mathrm{T} 1$ and $50 \%$ from $\mathrm{T} 0$ to $\mathrm{T} 2$ (mean values 14.8 at $\mathrm{T} 0 ; 10.1$ at $\mathrm{T} 1$; 
7.4 at T2) (Figure 2a). The DLQI more than halved within four weeks from 3.2 points to 1 point, indicating only a minor remaining reduction in life quality (Figure $2 b$ ). After four weeks of treatment (T1) the tolerability of the treatment was considered excellent according to $40 \%$ of subjects; good according to $50 \%$ of subjects, and fair according to $10 \%$ of subjects. No severe side effects were reported. Eight weeks of treatment reduced the amount of open and closed comedones (mean reduction of 12.5 comedones ( $80 \%$ reduction)) as well as inflammatory pustulae (mean reduction of 2.5 pustulae (65\% reduction)) resulting in an overall purer skin texture. Patient examples of improved acne disease are displayed in Figure $3 \mathrm{a}, \mathrm{b}$.

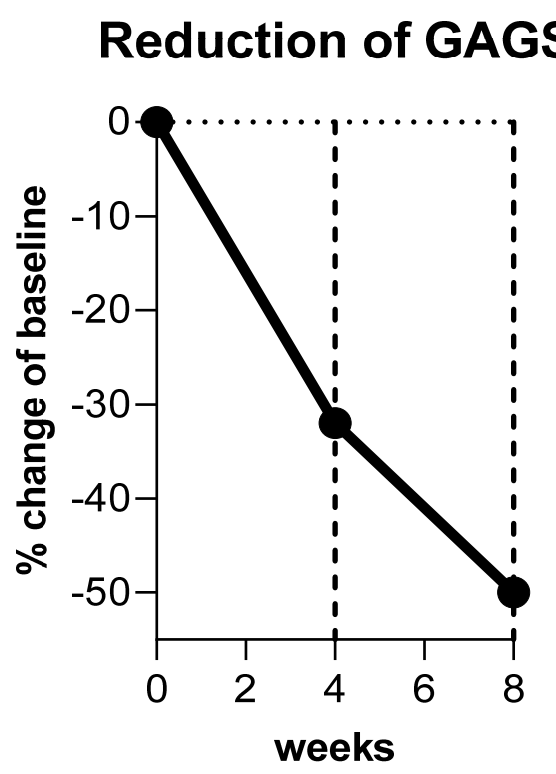

(a)

\section{Reduction of DLQI}

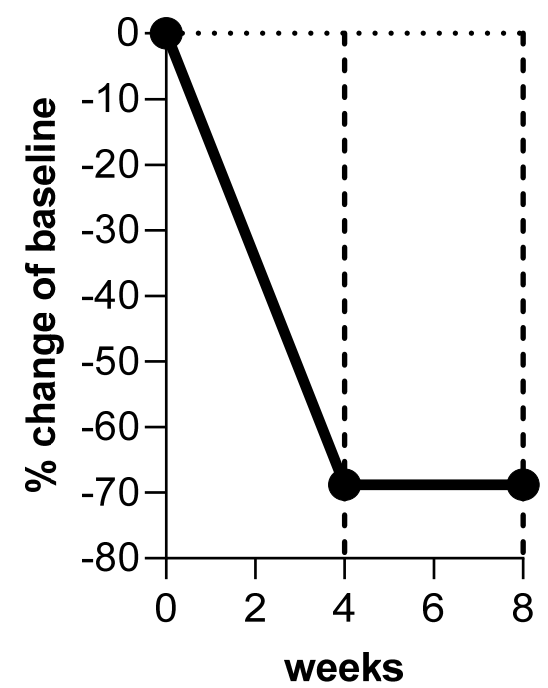

(b)

Figure 2. Clinical improvement of acne vulgaris. (a) The Global Acne Grading System (GAGS) score was reduced by $32 \%$ after eight weeks of nitric oxide (NO)-producing gel treatment. (b) The Dermatology Life Quality Index (DLQI) more than halved resulting in an approximately 70\% reduction, indicating only a minor remaining decreased quality of life after treatment with NOproducing gel formulation.

\subsection{Clinical Evaluation of a NO-Producing Serum as Cosmetic Agent}

The results of the efficacy questionnaire are shown in Table 1. Of note, $73 \%$ of the participants stated that the product provided immediate hydration, $80 \%$ of participants found that the skin appeared brighter, and $73 \%$ of participants found that wrinkles, if present, had decreased or softened. The cosmetic qualities were evaluated favorably, and $86 \%$ of participants liked the product overall. Application of the product visually reduced wrinkles and dry lines (Figure 4a). Furthermore, application over four weeks resulted in an improvement of structural integrity with an increase of elastic fibers and an increase of papillary dermis (Figure 4b) and smoothing of skin relief (Figure 4c). Overall changes are indicated in Figure 4d. No abnormal clinical signs were observed in any patient after four weeks of serum use. 


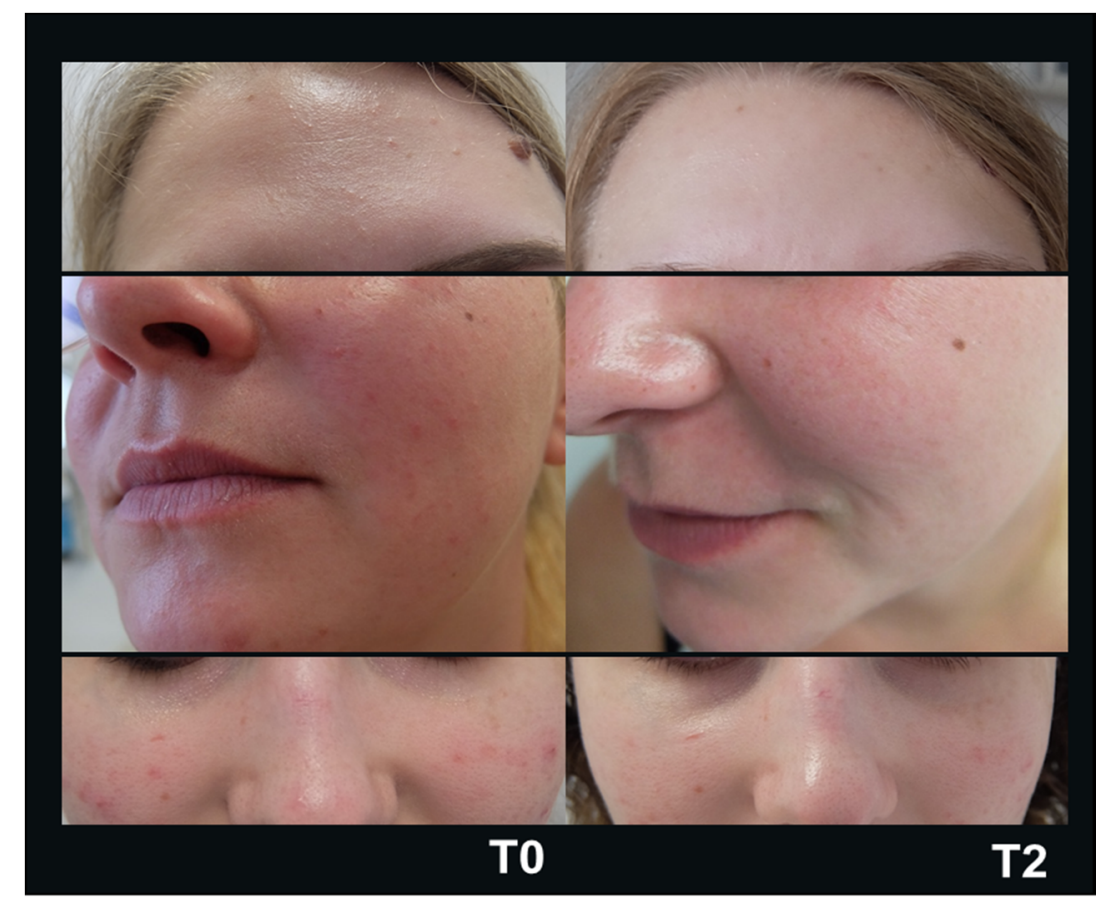

(a)

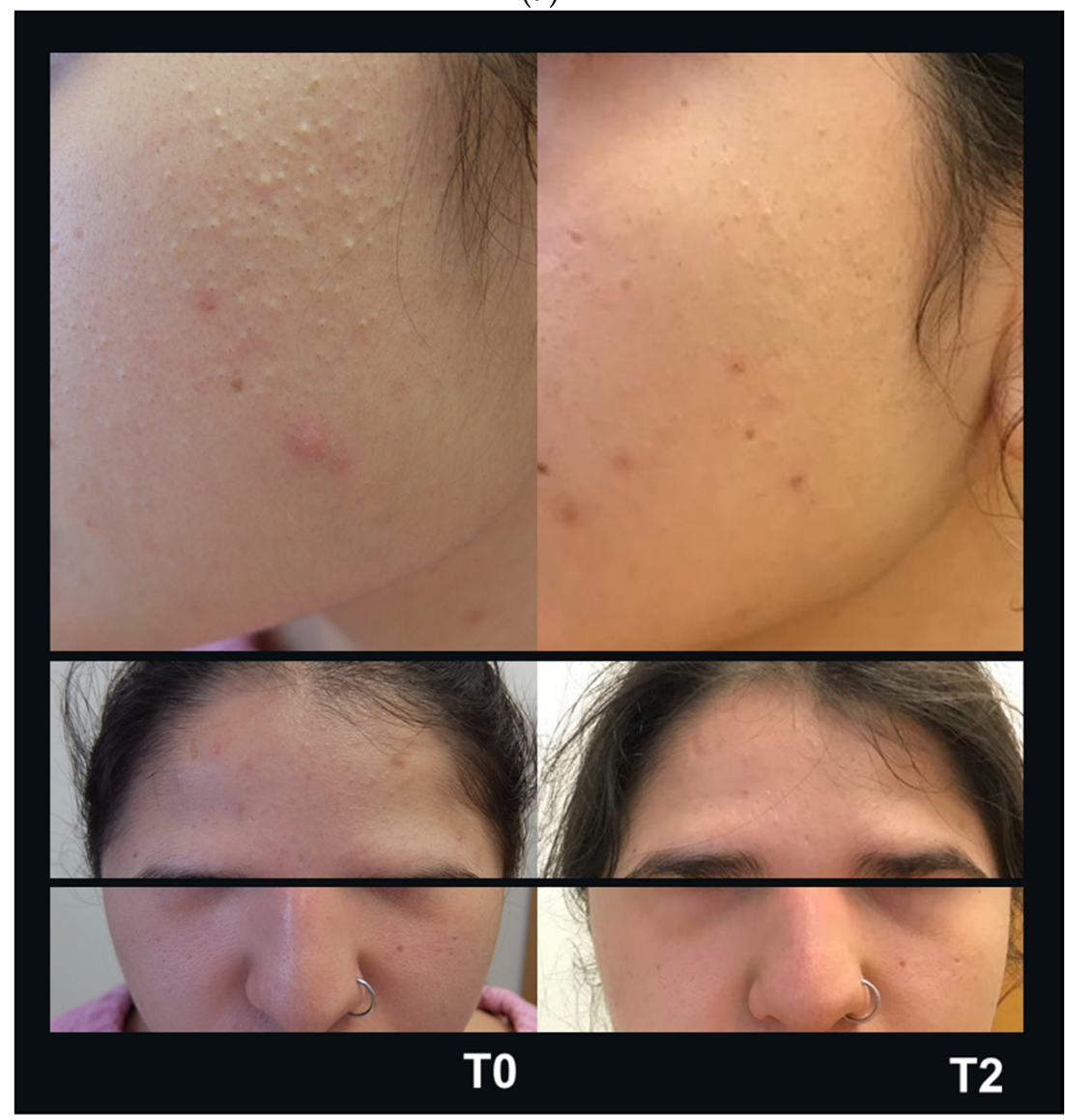

(b)

Figure 3. Improvement of acne vulgaris. Eight weeks of nitric oxide (NO)-producing gel formulation treatment (T2) reduced the amount of closed and open comedones and inflammatory pustulae in mild (a) and moderate (b) acne resulting in a purer skin texture. 


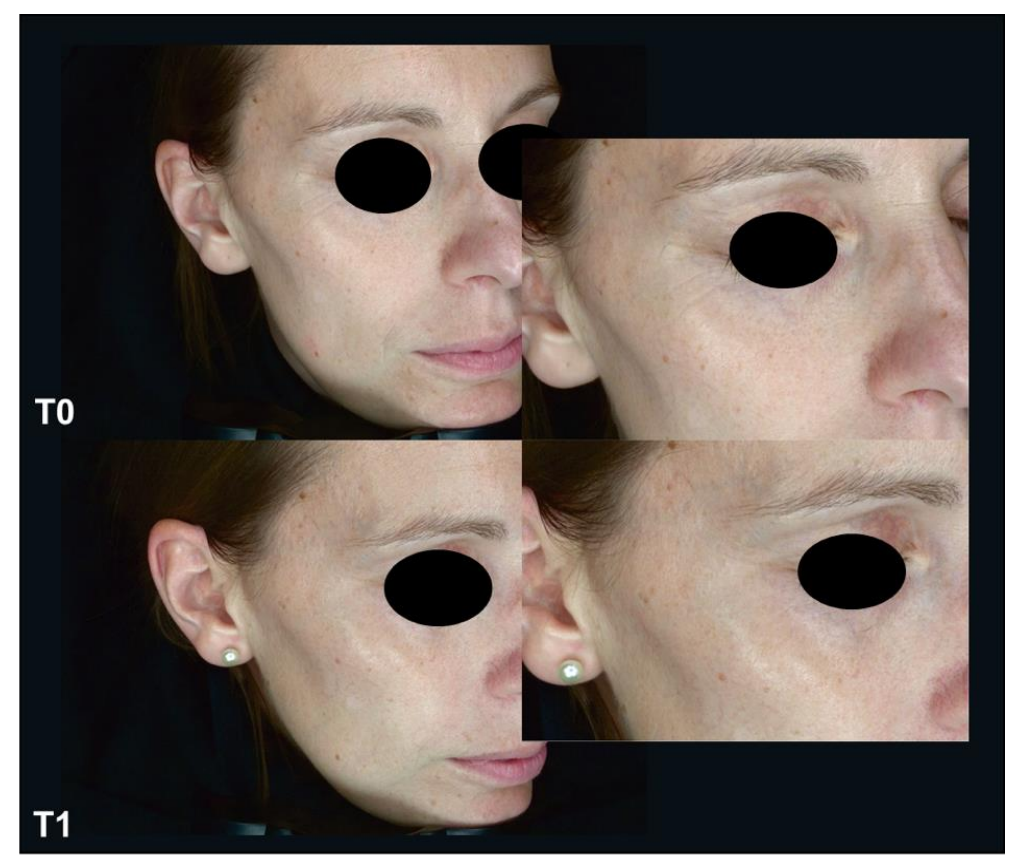

(a)
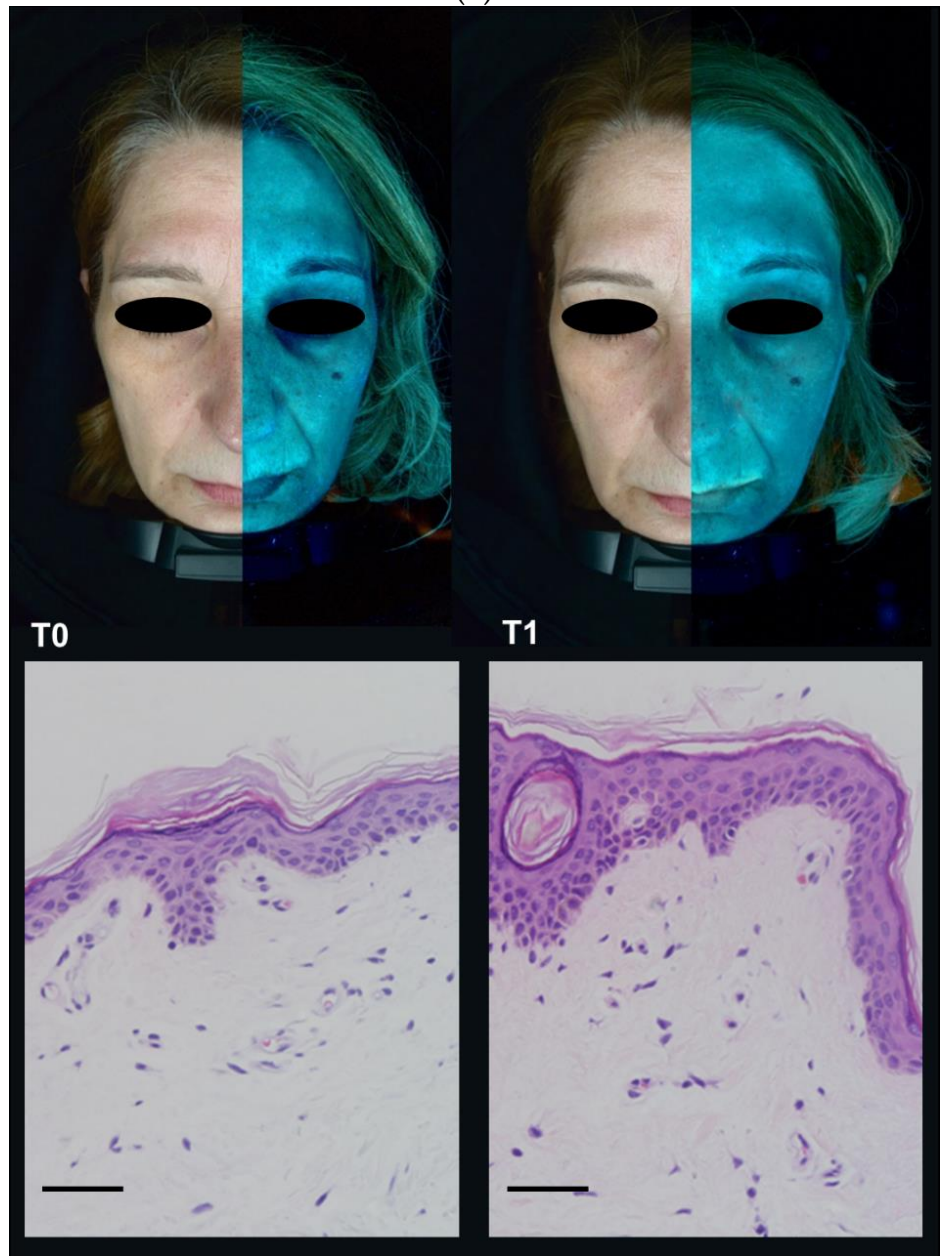

(b)

Figure 4. Cont. 


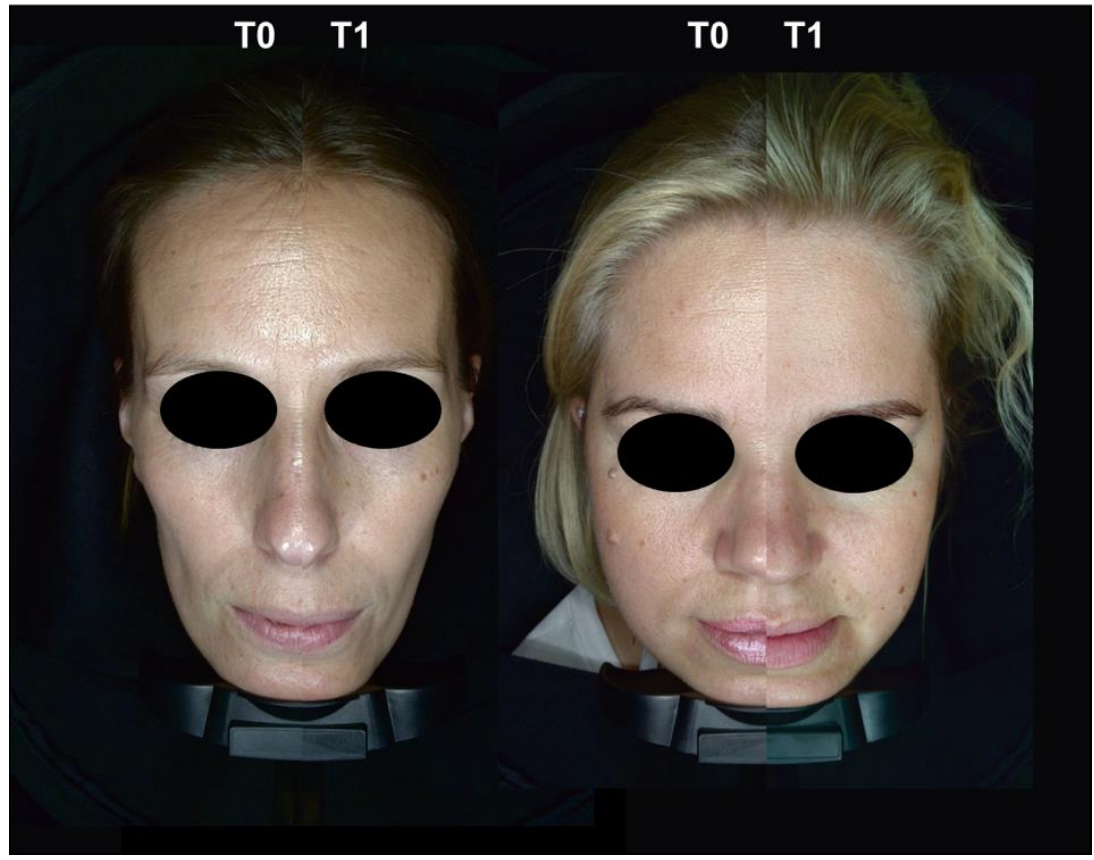

(c)

\section{Clinical evaluation of NO-serum as cosmetic agent}

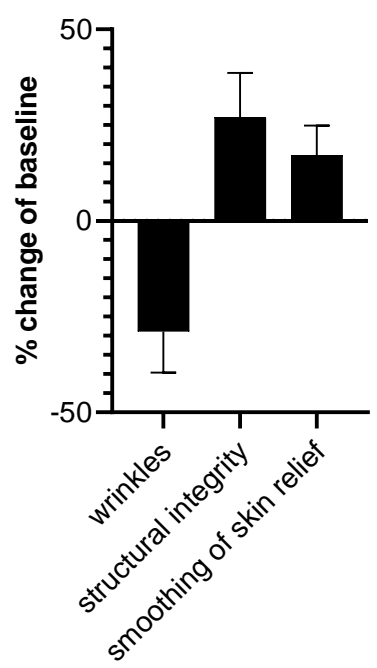

(d)

Figure 4. Clinical evaluation of nitric oxide (NO)-producing serum formulation as cosmetic agent. (a) Four weeks (T1) of application reduced wrinkles and dry lines significantly. (b) Four weeks of application furthermore increased structural integrity and led to (c) an overall smoothing of skin relief. Overall changes are displayed in (d). Scale bars: $50 \mu \mathrm{m}$.

Table 1. Results of the efficacy questionnaire.

\begin{tabular}{cc}
\hline Question of Efficacy Questionnaire & Percentage \\
\hline "I felt an improvement in skin hydration" & $73 \%$ \\
"My lines and wrinkles have softened (are less notable)" & $73 \%$ \\
"The skin of my face looks brighter" & $80 \%$ \\
"The texture of my skin is improved" & $86 \%$ \\
"I liked the product overall" & $86 \%$ \\
"I would like to continue using the product" & $86 \%$ \\
\hline
\end{tabular}




\section{Discussion}

Acne vulgaris of the face is one of the most encountered diseases in all dermatology and primary care practice and inherits a distinct disease burden with a high degree of psychosocial anguish, even in its mild form $[7,16]$. Although improvements in medical and nonmedical treatment have improved patients' outcomes in severe acne vulgaris [13], the treatment of mildly or moderately affected patients, that are not targets of aggressive systemic therapies, is still challenging. With its four key pathogenic factors of increased sebum production, alterations of the follicular keratinization processes, inflammation and colonialization of Cutibacterium acnes [8], a treatment should address as many of the pathogenic factors as possible. NO may here play a decisive role [3]. Our study clearly demonstrates an improvement in acne lesions over an eight-weeks treatment period. Using this novel NO-producing gel formulation, all patients experienced a significant reduction of both inflammatory and noninflammatory lesions. No one reported major side effects. The overall product tolerability was defined as "excellent" by $70 \%$ of the subjects at the end of the treatment. This indicates a lower therapeutic burden. The observed effects may be due to the antibiotic effects of $\mathrm{NO}$ and the inflammatory-modifying impact on even preclinical inflammation by modifying the $\mathrm{T}$ helper cells activity as well as the inhibition of inflammatory cytokines like IL- 8 and IL- 6 as well as TNF- $\alpha$ [3]. An inhibition of caspases by NO leads to a suppression of NLRP3 inflammasome activity and thereby reduces the expression of IL-1 $\beta$. NO furthermore reduces the T helper 17 cell activity via reduced expression of IL-17 and stimulation of regulatory T cells [17-20]. The effects of NO on the pathogenic factor of (hyper)keratinization are discussed controversially [20,21] Effects of NO on acne vulgaris [3,17-20] are summarized in Figure 5.

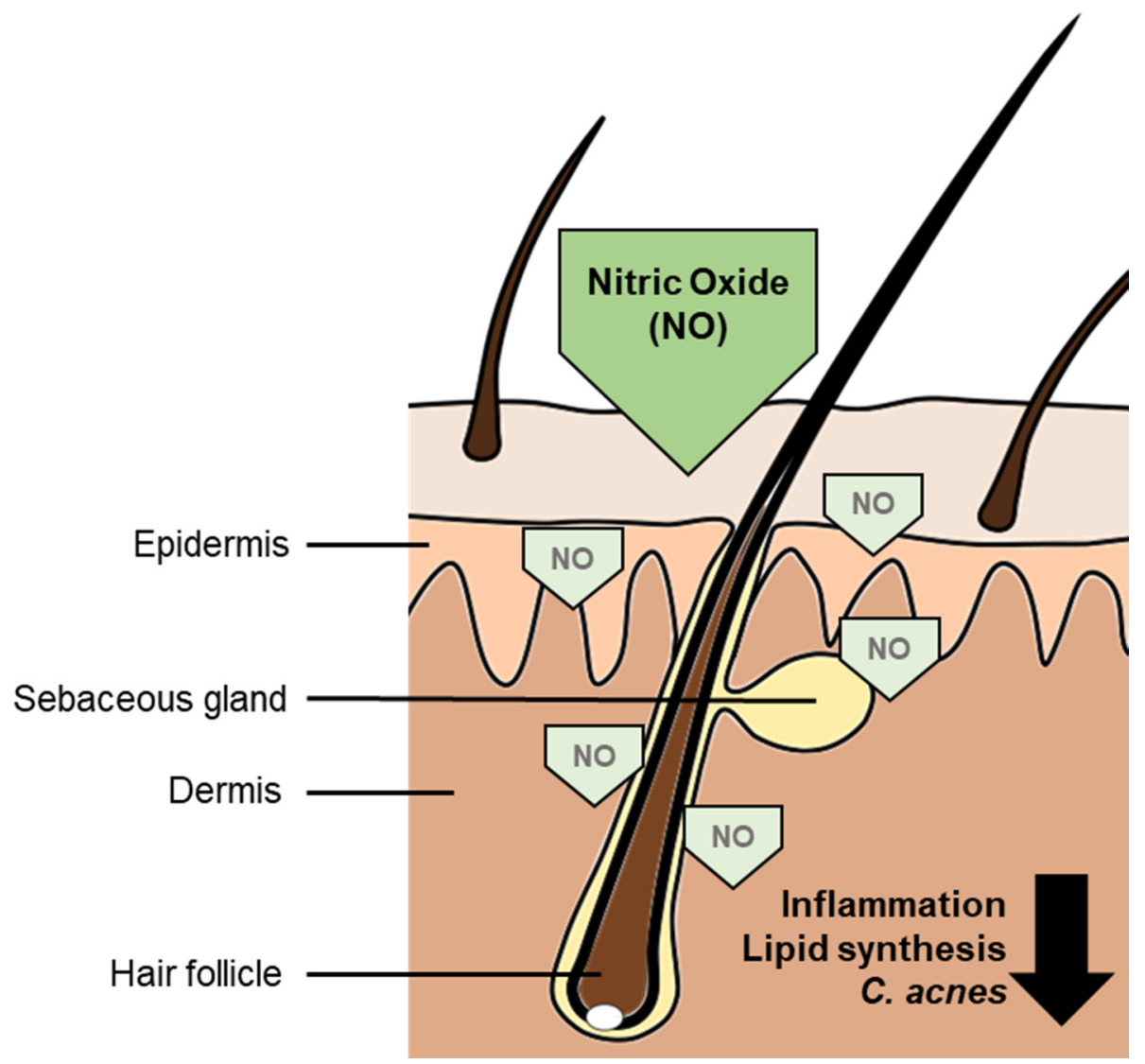

Figure 5. Disease-modifying effects of nitric oxide (NO) on acne vulgaris. The antimicrobial activity leads to a reduction of Cutibacterium acnes colonization. It furthermore reduces inflammation and lipid synthesis, contributing to an improvement of acne vulgaris disease. 
The promising results of the clinical evaluation as cosmetic agent point towards a favorable use in beautifying as well as maintaining and protective effects and may, due to its physiological functions, increase collagen synthesis in fibroblasts to decrease wrinkle formation [22]. With its novel dual component mutual-activating stable-release formulation we see benefits over other NO-generating formulations [6] It does not inherit the risk of methemoglobin formation like diazeniumdiolates, it is as easy to use as common gel- and serum-formulations and its applicability is not limited by technical limitations as seen in direct application of gaseous NO.

\section{Limitations of the Studies}

Of course, some limitations apply to our exploratory open-label pilot studies. Not designed with a placebo arm, the designs did not inherit a comparative trial of different therapeutic strategies in both studies, clinical evaluation for treatment of acne vulgaris as well as evaluation as a cosmetic agent. Eight weeks (acne vulgaris study) or four weeks (cosmetic evaluation) of observational period may be too short for long-term follow-up observation. Therefore, also because of the limited sample size, studies on larger patient populations should be performed. Nevertheless, the patient population as well as the proband population represent the common population presenting to general practitioners and dermatologists, resulting in a good external validity.

\section{Conclusions}

The NO gel-formulation is an innovative topical acne vulgaris treatment with a bimodal mode of action. It may perform its effects by inhibiting Cutibacterium agnes proliferation as well as restoring the skin's protective barrier and furthermore modifying inflammatory activity. Due to its excellent tolerability, it may reduce the therapeutic burden in acne vulgaris treatment. Furthermore, a NO-producing serum with increased fluidity seems to be beneficial in the aging skin population. Both novel formulations may exhibit benefits over other modes of application.

Author Contributions: Conceptualization, T.R., U.B.H.-C. and I.S.; methodology, I.S.; investigation, all authors; resources, T.R., U.B.H.-C. and I.S.; writing-original draft preparation, S.S. and I.S.; writing - review and editing, all authors; visualization, S.S. and I.S.; supervision, I.S. and T.R.; project administration, T.R. All authors have read and agreed to the published version of the manuscript.

Funding: This research received no external funding.

Institutional Review Board Statement: The study was conducted according to the guidelines for Good Clinical Practice [23] and the guidelines of the Declaration of Helsinki [24] and approved by the Institutional Ethics Committee of the Medical Faculty of the University of Duisburg-Essen (protocol code 20-9698-BO from 26 November 2020).

Informed Consent Statement: Informed consent was obtained from all subjects involved in the study.

Data Availability Statement: The data presented in this study are available on request from the corresponding author. The data are not publicly available due to data protection law.

Acknowledgments: We thank all participants for participation in the studies. We would like to thank Ricarda Ulankiewicz for her technical support, Fabrice Reyes for CLD measurements and Matz Dietrich for chemical advice.

Conflicts of Interest: The authors declare no conflict of interest.

\section{References}

1. Totzeck, M.; Hendgen-Cotta, U.B.; Rassaf, T. Nitrite-Nitric Oxide Signaling and Cardioprotection. Adv. Exp. Med. Biol. 2017, 982, 335-346. [CrossRef]

2. Rassaf, T.; Lauer, T.; Heiss, C.; Balzer, J.; Mangold, S.; Leyendecker, T.; Rottler, J.; Drexhage, C.; Meyer, C.; Kelm, M. Nitric oxide synthase-derived plasma nitrite predicts exercise capacity. Br. J. Sports Med. 2007, 41, 669-673. [CrossRef] [PubMed]

3. Del Rosso, J.Q.; Kircik, L.H. Spotlight on the Use of Nitric Oxide in Dermatology: What Is It? What Does It Do? Can It Become an Important Addition to the Therapeutic Armamentarium for Skin Disease? J. Drugs Dermatol. 2017, 16, s4-s10. [PubMed] 
4. Han, G.; Zippin, J.H.; Friedman, A. From bench to bedside: The therapeutic potential of nitric oxide in dermatology. J. Drugs Dermatol. 2009, 8, 586-594. [PubMed]

5. Kutner, A.J.; Friedman, A.J. Use of nitric oxide nanoparticulate platform for the treatment of skin and soft tissue infections. Wiley Interdiscip. Rev. Nanomed. Nanobiotechnol. 2013, 5, 502-514. [CrossRef]

6. Adler, B.L.; Friedman, A.J. Nitric oxide therapy for dermatologic disease. Future Sci. OA 2015, 1, FSO37. [CrossRef]

7. Zaenglein, A.L. Acne Vulgaris. N. Engl. J. Med. 2018, 379, 1343-1352. [CrossRef]

8. Pochi, P.E.; Shalita, A.R.; Strauss, J.S.; Webster, S.B.; Cunliffe, W.J.; Katz, H.I.; Kligman, A.M.; Leyden, J.J.; Lookingbill, D.P.; Plewig, G.; et al. Report of the Consensus Conference on Acne Classification. Washington, D.C., March 24 and 25, 1990. J. Am. Acad. Dermatol. 1991, 24, 495-500. [CrossRef]

9. Adityan, B.; Kumari, R.; Thappa, D.M. Scoring systems in acne vulgaris. Indian J. Dermatol. Venereol. Leprol. 2009, 75, 323-326. [CrossRef]

10. Hyun, E.; Bolla, M.; Steinhoff, M.; Wallace, J.L.; Soldato, P.D.; Vergnolle, N. Anti-inflammatory effects of nitric oxide-releasing hydrocortisone NCX 1022, in a murine model of contact dermatitis. Br. J. Pharmacol. 2004, 143, 618-625. [CrossRef]

11. Baldwin, H.; Blanco, D.; McKeever, C.; Paz, N.; Vasquez, Y.N.; Quiring, J.; Enloe, C.; De Leon, E.; Stasko, N. Results of a Phase 2 Efficacy and Safety Study with SB204, an Investigational Topical Nitric Oxide-releasing Drug for the Treatment of Acne Vulgaris. J. Clin. Aesthet. Dermatol. 2016, 9, 12-18.

12. Halvorsen, J.A.; Vleugels, R.A.; Bjertness, E.; Lien, L. A population-based study of acne and body mass index in adolescents. Arch. Dermatol. 2012, 148, 131-132. [CrossRef] [PubMed]

13. Garofalo, V.; Cannizzaro, M.V.; Mazzilli, S.; Bianchi, L.; Campione, E. Clinical evidence on the efficacy and tolerability of a topical medical device containing benzoylperoxide $4 \%$, retinol $0.5 \%$, mandelic acid $1 \%$ and lactobionic acid $1 \%$ in the treatment of mild facial acne: An open label pilot study. Clin. Cosmet. Investig. Dermatol. 2019, 12, 363-369. [CrossRef] [PubMed]

14. Tsai, M.C.; Chen, W.; Cheng, Y.W.; Wang, C.Y.; Chen, G.Y.; Hsu, T.J. Higher body mass index is a significant risk factor for acne formation in schoolchildren. Eur. J. Dermatol. 2006, 16, 251-253. [PubMed]

15. Finlay, A.Y.; Khan, G.K. Dermatology Life Quality Index (DLQI)—A simple practical measure for routine clinical use. Clin. Exp. Dermatol. 1994, 19, 210-216. [CrossRef]

16. Zaenglein, A.L.; Pathy, A.L.; Schlosser, B.J.; Alikhan, A.; Baldwin, H.E.; Berson, D.S.; Bowe, W.P.; Graber, E.M.; Harper, J.C.; Kang, S.; et al. Guidelines of care for the management of acne vulgaris. J. Am. Acad. Dermatol. 2016, 74, 945-973.e933. [CrossRef]

17. Aslam, I.; Fleischer, A.; Feldman, S. Emerging drugs for the treatment of acne. Expert Opin. Emerg. Drugs 2015, $20,91-101$. [CrossRef]

18. Hernandez-Cuellar, E.; Tsuchiya, K.; Hara, H.; Fang, R.; Sakai, S.; Kawamura, I.; Akira, S.; Mitsuyama, M. Cutting edge: Nitric oxide inhibits the NLRP3 inflammasome. J. Immunol. 2012, 189, 5113-5117. [CrossRef]

19. Kim, M.; Yin, J.; Hwang, I.H.; Park, D.H.; Lee, E.K.; Kim, M.J.; Lee, M.W. Anti-Acne Vulgaris Effects of Pedunculagin from the Leaves of Quercus mongolica by Anti-Inflammatory Activity and 5alpha-Reductase Inhibition. Molecules 2020, 25, 2154. [CrossRef]

20. Qin, M.; Landriscina, A.; Rosen, J.M.; Wei, G.; Kao, S.; Olcott, W.; Agak, G.W.; Paz, K.B.; Bonventre, J.; Clendaniel, A.; et al. Nitric Oxide-Releasing Nanoparticles Prevent Propionibacterium acnes-Induced Inflammation by Both Clearing the Organism and Inhibiting Microbial Stimulation of the Innate Immune Response. J. Investig. Dermatol. 2015, 135, 2723-2731. [CrossRef] [PubMed]

21. Mendes, D.A.; Horinouchi, C.D.; Prudente Ada, S.; Soley Bda, S.; Assreuy, J.; Otuki, M.F.; Cabrini, D.A. In vivo participation of nitric oxide in hyperproliferative epidermal phenomena in mice. Eur. J. Pharmacol. 2012, 687, 1-8. [CrossRef] [PubMed]

22. Jariashvili, K.; Madhan, B.; Brodsky, B.; Kuchava, A.; Namicheishvili, L.; Metreveli, N. UV damage of collagen: Insights from model collagen peptides. Biopolymers 2012, 97, 189-198. [CrossRef] [PubMed]

23. European Medicines Agency. ICH E6 (R2) Good Clinical Practice. 2016. Available online: https:/ / www.ema.europa.eu/en/iche6-r2-good-clinical-practice (accessed on 30 December 2020).

24. World Medical Association. World Medical Association Declaration of Helsinki: Ethical principles for medical research involving human subjects. JAMA 2013, 310, 2191-2194. [CrossRef] [PubMed] 